\title{
Avaliação de Chats com o uso de Learning Vectors - LVs
}

\section{(Chats Evaluation with the use of Learning Vectors - LVs)}

Eliana Moreira de Oliveira - Universidade Aberta do Brasil/CEFETCE eaufcmidias@gmail.com

Gilvandenys Leite Sales - Centro Federal de Educação Tecnológica do Ceará denyssales@cefetce.br

Giovanni Cordeiro Barroso - Universidade Federal do Ceará gcb@fisica.ufc.br

José Marques Soares - Centro Federal de Educação Tecnológica do Ceará marques@cefetce.br

Eveline Porto Sales Aguiar - Universidade Aberta do Brasil/CEFETCE evelineps@gmail.com

Resumo. Estabelecer juízo de valor de forma contínua e formativa em atividades a distância desenvolvidas de forma síncrona, tais como em interações de chats é o propósito deste artigo. Empregou-se uma metodologia de avaliação que faz uso do instrumento Learning Vectors (LVs) no ambiente virtual de aprendizagem MOODLE. Pretende-se com este trabalho ampliar as possibilidades de avaliação on-line em salas de chats de ambientes virtuais de educação a distância.

Palavras-chave: Educação a distância, Metodologia de Avaliação em Chats, Learning Vectors.

Abstract. Establish judge of value on a continuous and formative activities in the distance developed synchronously, such as interactions of chat is the purpose of this article. We applied a methodology to measure that makes use of the indicators of learning Learning Vectors (LVs) in the virtual learning environment Moodle. It's aiming with this work to expand the possibilities for evaluating online chats at the e-learning virtual environments.

Keywords: e-learning, Chats evaluation methodology, Learning Vectors.

\section{Introdução}

Como efetivação de políticas públicas de educação a distância traçadas pelo governo federal foi implantada a Universidade Aberta do Brasil (UAB) (Brasil, 2006), cujos cursos na modalidade semipresencial têm por meta formar docentes nas mais diversas áreas dos saberes para suprir a demanda da falta de professores explicitadas por dados estatísticos do INEP (Brasil, 2003).

Tal medida tem levado as instituições de ensino superior envolvidas a fazer uso de Ambientes Virtuais de Aprendizagem (AVA) e lidar com suas ferramentas síncronas e assíncronas de interação.

Colaborando com este processo esta pesquisa tem por propósito aplicar e validar instrumentos de avaliação que se agreguem a esses ambientes, particularmente em ferramentas síncronas como Chats, ou salas de bate-papo.

A mesma desenvolve-se como parte de um projeto que valida ferramentas para o portal da Educação Profissional e Tecnológica (PORTAL EPT, 2008) e se propõe a 
colaborar com a educação a distância (EaD), por meio da inclusão de instrumentos semi-automatizados de avaliação qualitativa nos AVA.

\section{Aprendizagem a Distância: pressupostos e avaliação}

Algumas características devem ser observadas na aprendizagem a distância: colaboração, interação e autonomia.

Colaboração que vise a formação de uma comunidade de aprendizes com foco na aprendizagem coletiva, quando se trata de adquirir conhecimento, segundo Freire (1981, p. 71): "Conhecer que é sempre um processo, supõe uma situação dialógica. Não há, estritamente falando, um 'eu penso', mas um 'nós pensamos"”.

Interação dada pela troca de mensagens no AVA, ou mesmo pelo diálogo presencial, permitidos no modelo semi-presencial da UAB é outro aspecto fundamental na aprendizagem, afinal ao se criar situações dialógicas permite-se a externalização de idéias e a formação de esquemas de aprendizagem.

Incitar a interação e a colaboração podem contribuir na formação de um aprendiz autônomo, gestor de seu tempo e de sua aprendizagem.

Para colaborar com a autonomia de um aprendiz deve-se oferecer caminhos para que ele possa administrar seu ritmo e regular sua aprendizagem, desta forma, indicadores de sua aprendizagem e instrumentos de avaliação que promovam feedbacks rápidos serão de fundamental importância.

Com relação à avaliação, Zabala (1998) expressa que sua finalidade é: “(...) ser um instrumento educativo que informa e faz uma valoração do processo de aprendizagem seguido pelo aluno, com o objetivo de oportunizar, em todo momento, as propostas educacionais mais adequadas". Segundo Perrenoud (1999) ela deve ser formativa e acompanhar todo processo possibilitando a regulagem da aprendizagem.

Ambientes virtuais atualmente em uso, a saber: o AulaNet da PUC-Rio (Gerosa et al., 2004; Escovedo et al., 2006), TelEduc da UNICAMP (Otsuka e Rocha, 2005; Rocha, 2006), ROODA da UFRGS (Bassani, 2004; Bassani e Behar, 2005), o eProinfo/MEC e SOLAR/UFC, têm procurado oferecer um modelo alinhado ao paradigma da colaboração, interação, autonomia e uma avaliação contínua e formativa, assim como seus pesquisadores têm buscado otimizar e propor ferramentas que possibilitem adequação a este modelo.

Para estes ambientes encontram-se muitas propostas de avaliação e implementações para ferramentas de interação do tipo fóruns de discussão seja de natureza técnica e/ou metodológica (Araújo, 2005; Otsuka e Rocha, 2005; Lopes, 2007; Gerosa et al., 2004; David et al., 2007). Entretanto, quando se trata de avaliação online de alunos em chats a literatura apresenta-se ainda de forma escassa, limitando-se a análise das interações nas trocas de mensagens e das potencialidades da ferramenta chat (Lachi, 2003; Pereira, 2004; Pimentel, 2003 e 2004).

Para avaliar alunos em Chats ainda não se desenvolveu um instrumento que efetivamente proporcione uma avaliação qualitativa e formativa em $\mathrm{EaD}$, que, além de mediar, possibilite também regular aprendizagens em tempo real. Esta possibilidade é o que será abordado a seguir.

\section{Learning Vectors (Vetores Aprendizagem)}

Os Learning Vectors (LVs) (Sales, 2008 a, b) são indicadores de aprendizagem desenvolvidos para auxiliar o processo de avaliação em AVA. Sua implementação, 
testes e refinamentos estão sendo feitos no ambiente MOODLE do Centro Federal de Educação Tecnológica do Ceará (CEFETCE) em turmas da UAB.

Como instrumento de avaliação online os LVs reúnem aspectos qualitativos e quantitativos no acompanhamento de desempenho do aprendiz ao possibilitarem feedbacks constantes de suas ações. Outra característica dos LVs é reduzir a sobrecarga de trabalho gerada para o tutor.

As inserções do aluno e participação no curso, serão categorizadas segundo uma escala Likert-icônica (Figura 1):

\begin{tabular}{|c|c|}
\hline $\begin{array}{c}\text { LV } \\
\text { Ícone }\end{array}$ & CATEGORIZAÇÃO DAS MENSAGENS/ARQUIVOS ANEXADOS \\
\hline & $\begin{array}{l}\text { Muito Bom } \\
\text {-Reflexões aprofundadas; Interações/Postagens por meio de "Mensagens/Arquivos anexados" que podem apresentar, além } \\
\text { de respostas e comentários, significativos questionamentos, ou mesmo, sinteses de idéias que surgem na } \\
\text { discussão/aprendizagem do tema proposto, gerando mais interaçôes. }\end{array}$ \\
\hline & $\begin{array}{l}\text { Bom } \\
\text { - Boas reflexões; Interações/Postagens por meio de "Mensagens/Arquivos anexados" que apresentam respostas e } \\
\text { comentários significativos para discussão/aprendizagem do tema proposto. }\end{array}$ \\
\hline & $\begin{array}{l}\text { Regular } \\
\text { - Razóveis reflexões; Interações: Postagens por meio de "Mensagens/Arquivos anexados" ainda limitados e de forma } \\
\text { superficial acerca do tema proposto para a discussão/aprendizagem. }\end{array}$ \\
\hline & $\begin{array}{l}\text { Fraco } \\
\text { - Reflexões vazias de conteúdo; Interações/Postagens por meio de "Mensagens/Arquivos anexados" que pouco agregam } \\
\text { valor à discussão/aprendizagem do tema proposto. }\end{array}$ \\
\hline & $\begin{array}{l}\text { Não Satisfatório } \\
\text {-Aprendiz que assume posição passiva; Interações/Postagens por meio de "Mensagens/Arquivos anexados" que em nada } \\
\text { contribuem para a discussãolaprendizagem do tema proposto limitando-se a enviar mensagens de concordância ou arquivos } \\
\text { anexados sem originalidade. }\end{array}$ \\
\hline & $\begin{array}{l}\text { Mensagens/Arquivos anexados não avaliados } \\
\text {-Não se fez presente. } \\
\text {-Mensagens de esclarecimentos, saudações, recados, e agradecimentos. }\end{array}$ \\
\hline
\end{tabular}

Figura 1 - Categorização de Mensagens

Ao atribuir um destes LVs Ícones à mensagem do aluno, somente este a visualizará, garantindo assim a sua privacidade.

Durante o processo de avaliação o aluno poderá consultar seu LVômetro (Figura 2), que consta de uma representação geométrica onde a projeção horizontal do LV, vetor azul, relaciona-se à positividade de desempenho e a projeção vertical do LV à negatividade de desempenho, representadas, respectivamente pelas faixas verde e vermelha.

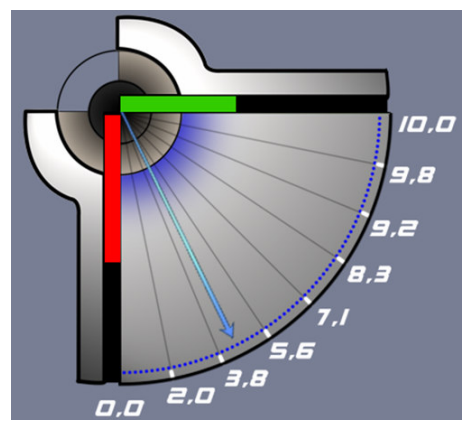

Figura 2 - LVômetro

À relação entre a positividade de desempenho e a negatividade de desempenho relaciona-se o fator qualitativo beta, cujo valor máximo é 6 .

\section{1 - O LV Chat}


O Chat LV constitui uma ferramenta síncrona que pode possibilitar avaliações online de um tema ou tópico previamente enviado pelo tutor, diferentemente de uma sala de batepapo livre.

As mensagens postadas pelos alunos farão o LV Chat variar sua direção segundo a seguinte distribuição (Figura 3), em que $\alpha=7,5^{\circ}$ é um ângulo definido empiricamente:

\begin{tabular}{|c|c|c|}
\hline LVicone & $\begin{array}{c}\text { Valor } \\
\text { Associado }\end{array}$ & $\begin{array}{c}\text { Variacão } \\
\text { Angular } \\
\Delta\end{array}$ \\
\hline$\Theta$ & 0 & 0 \\
\hline$\because$ & 1 & $0,5 \alpha$ \\
\hline$\because$ & 2 & $1,0 \alpha$ \\
\hline$\because$ & 3 & $1,5 \alpha$ \\
\hline$\ddots$ & 4 & $2,0 \alpha$ \\
\hline
\end{tabular}

\section{Figura 3 - Variação Angular do LV Chat}

Para modelar o LV Chat, cuja fundamentação é baseada em um ente matemático vetorial, usou-se a seguinte expressão para a projeção horizontal do vetor (Equação 1):

Em que:

$$
N C_{k n}=10 * \cos \left[(-12 \alpha)+I_{n}\right]
$$

$\mathrm{NC}_{\mathrm{kn}}$ - Nota de cada Chat;

$\mathrm{k}=1,2,3 \ldots \mathrm{z}$; é o número de chats em discussão, sendo $\mathrm{z}$ o número total de chats

previstos no curso;

$\mathrm{n}=1,2,3 \ldots$ representa o número de mensagens postadas por parte do aluno;

$\alpha=7,5^{\circ}$ é o passo;

In é o parâmetro que mede a variação angular do vetor (limitado a $12 \alpha$ ), correlaciona-se às interações do aluno (Equação 2):

-Para $\mathrm{n}=0$ (Condição Inicial): $\mathrm{I}_{\mathrm{n}}=0$;

-Para $1 \leq \mathrm{n} \leq 4$ :

$$
I_{n}=\frac{1}{2} * L V * \alpha+I_{n-1}
$$

- A variável LV é função do LV Ícone escolhido e pode assumir os valores: $0,1,2,3$ ou 4, que estão associados aos LV Ícones (Figura 3).

-Para $\mathrm{n} \geq 5$, foi criado um fator penalizador (Equação 3), caso seja atribuido um LV Ícone vermelho:

$$
I_{n}=\left\{\begin{array}{l}
-0,5 \alpha+I_{n-1} ; \text { se } L V=0 \\
\frac{1}{2} * L V * \alpha+I_{n-1} ; \text { se } L V=1,2,3 \text { ou } 4 .
\end{array}\right.
$$

- Para limitar a variação angular do vetor em $90^{\circ}$, estabeleceu-se um limite para $I_{n}($ Equação 4):

$$
I_{n}=\left\{\begin{array}{l}
I_{n} ; \text { se } I_{n} \leq 12 \alpha \\
12 \alpha ; \text { se } I_{n}>12 \alpha
\end{array}\right.
$$

A partir da modelagem acima exposta elaborou-se algo mais didático para apresentação aos alunos com fins de esclarecer os critérios de avaliação nas interações no Chat Lv (Figura 4) para que ficasse compreensível ao aluno. O conceito de ponto está relacionado à separação entre duas marcas brancas no LVômetro. 


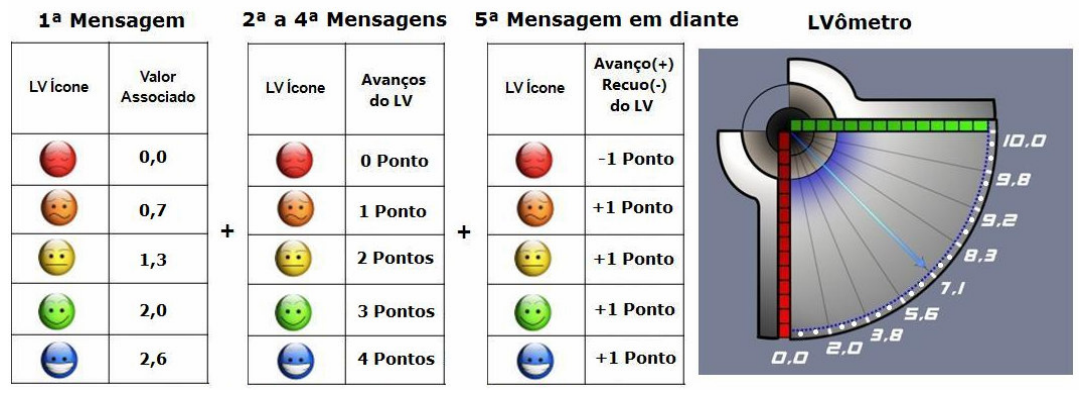

Figura 4 - Notas em Chats LV

Para exemplificar, seja um aluno que inicia um Chat LV com LV Ícone Verde (2,0 na nota), a seguir recebe uma seqüência de LVs Ícones Amarelo(mais 2 pontos), Azul (mais 4 pontos) e Verde (mais 3 pontos), sua nota avança 9 pontos, resultando em 7,1. A seguir recebe três LV Ícones Vermelhos (penalização de 3 pontos) em suas próximas interações, terá sua nota final reduzida em 3 pontos e ficará igual a 5,6.

Desenvolvido em PHP como parte de um módulo que se acopla ao ambiente virtual MOODLE, passou-se a fase de sua validação. A seção a seguir trata da aplicação do Chat LV.

\section{Metodologia e Análise de Resultados}

Aplicou-se o Chat LV a 16 turmas dos cursos de Matemática e Hotelaria da Universidade Aberta do Brasil (UAB) do Centro Federal de Educação Tecnológica do Ceará (CEFETCE) na disciplina de Introdução à EAD. O intuito não foi analisar as interações entre participantes, mas sim observar o comportamento da ferramenta Chat LV. Para tanto, tomou-se uma destas turmas e selecionou-se uma de suas sessões.

No planejamento da sessão de Chat LV solicitou-se que os alunos fizessem uma leitura de um texto previamente enviado, que seria utilizado para nortear as discussões, bem como orientou-se que entrassem antes do horário marcado para os mútuos cumprimentos.

Durante a realização do Chat LV, que teria 60 minutos de duração, sugeriu-se tratar de assuntos exclusivos do texto, uma vez que os questionamentos e o debate referir-se-iam apenas ao tema proposto.

Para um aproveitamento maior da sessão de Chat LV, a turma foi dividida em grupos de 10 alunos.

$\mathrm{Na}$ tentativa de focar o bate-papo em torno do tema proposto, as palavras iniciais do tutor foram: "Em primeiro lugar antes de iniciar o chat, quero mencionar alguns pontos importantes: Estamos aqui para tratar do texto, não vamos utilizar este momento para falar de assuntos pessoais com os colegas. E o chat irá funcionar com os debates que eu irei propor, ao passo que eu for levantando questionamentos, vocês expõem seu ponto de vista, ok? Assim funciona bem melhor".

O papel do tutor foi de mediar, instigar e questionar. Abaixo, um trecho na íntegra, de um bate-papo realizado em uma das turmas:

Tutor - "A aprendizagem não se faz só na sala de aula, mas em inúmeros espaços de encontro, como o fórum, por exemplo. Como vocês analisam o aprendizado de vocês neste ambiente?"

Aluno 1 - "Os fóruns nos direciona pensamentos, com o auxilio dos colegas enriquecemos um pouco mais o nosso conheco nosso conhecimento". 
Aluno 2 - "O aprendizado nessa nova maneira tem sido sim bastante interessante, dispertando em mim curiosodades e dai começo a exploram mais as ferramentas usadas".

Aluno 3 - "acho que estamos interagindo bem nos foruns".

Aluno 4 - "Tenho aprendido muito com os colegas e com voce atraves do ambiente".

Aluno 5 - "Devido o curso ser de boa qualidade o fórum me motiva muito em participar, questionar juntos com os colegas sobre o assunto proposto, essa intração é muito importante para adquirir mais conhecimentos".

No transcorrer do Chat LV o tutor, à proporção que avalia as mensagens, deve procurar clicar nos LV Ícones, o sistema apresenta cada aluno com seu número de interações e permite que se consulte sua nota em "visualizar LV" (Figura 5). Para garantir a privacidade da avaliação, cada aluno só pode visualizar sua própria nota, assim como, só enxerga os seus LV ícones.

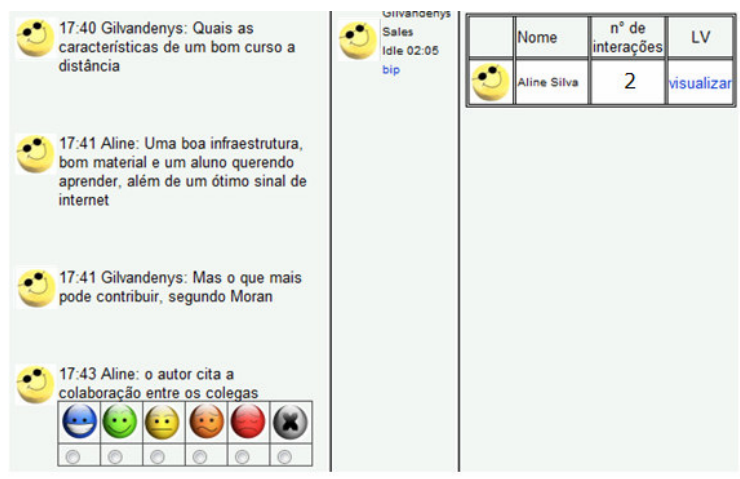

Figura 5 - Tela do MOODLE vista pelo tutor

O aluno no decorrer do chat pode acompanhar a avaliação que está sendo atribuída pelo tutor a cada mensagem postada (Figura 6), bem como observar a sua quantidade e a nota associada aos respectivos LVs Ícones que foram selecionados.

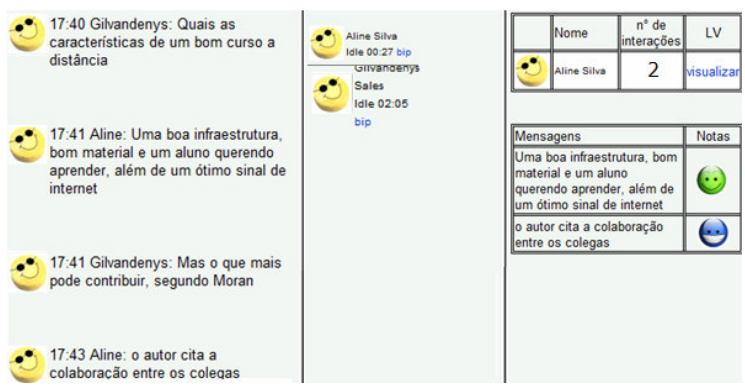

Figura 6 - Tela do MOODLE vista pelo tutor

Ao clicar em "visualizar LV", o aluno verifica sua situação no LVômetro (Figura 7): número de interações de cada LV Ícone, nota obtida e o fator qualitativo beta. Estes dados permitem o acompanhamento de seu crescimento no Chat LV e podem ajudar a melhorar a qualidade de suas mensagens. 


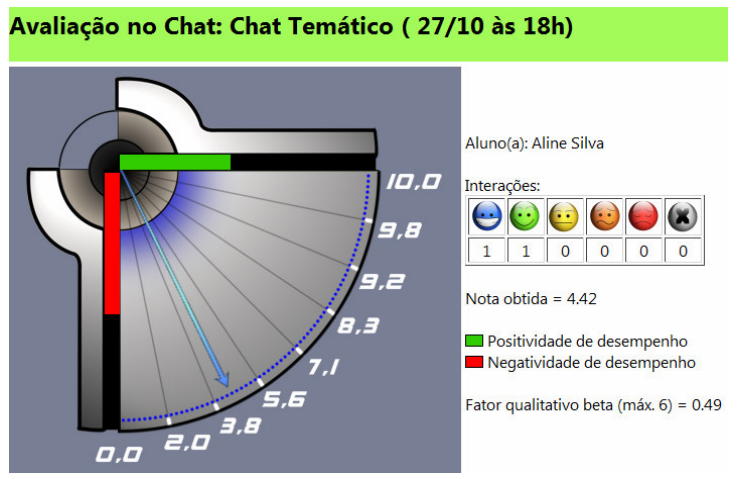

Figura 7 - Visualizar LVômetro

$\mathrm{Na}$ avaliação feita pelo tutor nessa sessão de chat os resultados foram satisfatórios, uma vez que as notas obtidas por 08 alunos estão entre um intervalo de 8,66 e 10,0. Entretanto, um aluno não atingiu bom resultado, pois obteve nota igual a 2,59, face sua pouca interação na sala de Chat LV.

Ressalta-se que é possível os alunos melhorarem seu rendimento no Chat LV, pois quanto mais interações de boa qualidade ele enviar, maiores serão suas chances de obter uma melhor nota.

Durante a realização do Chat $\mathrm{LV}$, percebeu-se que por ser uma ferramenta síncrona muito dinâmica houve dificuldades em sua avaliação online, fato apresentado pelos tutores das 16 turmas. Portanto, no transcorrer de uma sessão de Chat LV pode-se optar pela liberação ou não do LVômetro deixando para um momento posterior a sua divulgação.

Durante a sessão de Chat LV os LVs Ícones podem aparecer para o aluno, uma vez que o tutor consiga assinalá-los durante a sua realização, as demais mensagens podem ser avaliadas off-line.

A seguir tecem-se nossas considerações finais e levantam-se pressupostos para trabalhos futuros.

\section{Conclusões}

O Chat LV, a priori idealizado para ser utilizado online, pode constituir-se como uma ferramenta que possibilita uma avaliação contínua e formativa, uma vez que o tutor consiga fazer a seleção dos LV Ícones ainda durante a sua realização, ação esta que oportuniza ao aluno regular sua aprendizagem e motivá-lo a cada vez mais interagir com os demais no ambiente virtual.

Frente a atividades em que o aluno saiba que está sendo avaliado considera-se a dinâmica do Chat LV um momento para que o tutor possa analisar o seu comportamento de forma síncrona, uma vez que se pode observar como colaboram e interagem a partir de suas mensagens em torno do tema proposto.

Espera-se que este trabalho possa ampliar as possibilidades de avaliação on-line em salas virtuais.

Pretende-se em trabalhos futuros desenvolver agentes inteligentes com o propósito de agrupar automaticamente a classificação realizada pelo professor-tutor, resultando em uma pré-classificação por cores de LV Ícones para subsidiar a análise de interações em chats. Os agrupamentos apresentados irão auxiliar o professor-tutor a avaliar a qualidade das mensagens, bem como se as inserções dos alunos foram satisfatórias ou não, frente às discussões propostas para o chat. 


\section{Referências}

Araújo, L. H. L. e Filho, G. J. L. Comunidades virtuais de aprendizagem: novas dinâmicas de aprendizagem exigem novas formas de avaliação. In: Simpósio Brasileiro sobre Informática na Escola. 2005.

Bassani, P. Modelagem e mapeamento das interações em um ambiente virtual de aprendizagem. Projeto de Tese de doutorado, Pós-Graduação em Informática na Educação/UFRGS, 2004.

Bassani, P. B. S.; Behar, P. A. interROODA: mapeamento das interações individuais e interindividuais no ambiente virtual de aprendizagem ROODA. In: RENOTE Revista Novas Tecnologias na Educação, v.3, n2. Porto Alegre, 2005.

Brasil 2003. Estatística dos Professores no Brasil - Outubro de 2003. (online). Disponível em: < http://www.inep.gov.br/estatisticas/professor2003/ >. Acesso em: 20/06/2008.

Brasil 2006. Decreto $\mathbf{N}^{\mathbf{5}} \mathbf{5 6 2 2}$ de 19/12/2005. (online). Disponível em: $<$ http://portal.mec.gov.br >. Acesso em: 20/06/2007.

David, P. B.; Pequeno, M. C.; Silva, A. S. R. da.; Souza, C. F.; Júnior, G. S. V.; Castro Filho, J. A. de; Ventura, P. P. B.; Maia, S. M. Avaliação da Aprendizagem em Educação a Distância numa Perspectiva Sócio-Interacionista. In: Anais do XVIII SBIE - Simpósio Brasileiro de Informática na Educação, 2007. São Paulo - SP.

EPT VIRTUAL. Portal da Educação Profissional e Tecnológica. Ministério da Educação. (online). Disponível em: < http://eptad.cefetce.br>. Acesso em: 20 Out 2008.

Freire, P. Ação cultural para a liberdade. $5^{\text {a }}$ ed., Rio de Janeiro, Paz e Terra. 1981.

Gerosa, M. A., Fuks, H. e Lucena, C. J. P. Estruturação e categorização de mensagens em ferramentas de comunicação textuais assíncronas. In: WORLD CONGRESS ON ENGINEERING AND TECHNOLOGY EDUCATION - WCETE'2004, Santos Proceedings... Santos: COPEC/IEEE, 2004.

Lachi, R. L. Um Agente de Interface para Ferramentas de Bate-Papo em Ambientes de Ensino a Distância na Web. Dissertação de Mestrado, IC/Unicamp, 2003.

Lopes, M. S. S. Avaliação da aprendizagem em atividades colaborativas em EaD viabilizada por um fórum categorizado. Universidade Federal do Rio de Janeiro, Instituto de Matemática, Núcleo de Computação Eletrônica. Rio de Janeiro, 2007. 168 f. Dissertação de Mestrado.

Pereira, V. O. Bate-Papo na Internet: algumas perspectivas educativas. Programa de Pós-Graduação em educação Brasileira, UFC. Dissertação de Mestrado. Fortaleza, 2004.

Perrenoud, P. Avaliação: da excelência à regulação das aprendizagens - entre duas lógicas. Porto Alegre: Artmed Editora, 1999.

Pimentel, M. G.; Fuks, H.; Lucena, C. J. P. Avaliação da Participação dos Aprendizes em Debates Síncronos. In XIV Simpósio Brasileiro de Informática na Educação SBIE., NCE-UFRJ, Rio de Janeiro, 2003.

Pimentel, M. G., Fuks, H., Lucena, C. J. P. Engenharia de Groupware no desenvolvimento de uma ferramenta de bate-papo para debates educacionais: diminuindo a confusão da conversação, XV Simpósio Brasileiro de Informática na Educação - SBIE/SBC, 9- 12 Novembro, 2004, ISBN 85-7401-161-4, Manaus AM, pp 149-157. 
Otsuka, J. L. e Rocha, H. V. Um modelo de suporte à avaliação formativa para ambientes de EaD. Campinas: Instituto de Computação-Unicamp. (Relatório Técnico IC-05-11). 2005.

Rocha, V. et al. Avaliação online: o modelo de suporte tecnológico do projeto Teleduc. In: SILVA, Marco; SANTOS, Edméa (Org.). Avaliação da aprendizagem em educação online. São Paulo: Loyola. 2006, p. 347-368.

Sales, G. L. ; Barroso, G. C. ; Soares, J. M. O Indicador de Aprendizagem Learning Vectors Como Instrumento Automatizado de Avaliação para Suporte à Aprendizagem em EaD. In: Workshop Sobre Informática na Escola - WIE, 2008, Belém, v. 1. p. 205-214.

Sales, G. L. ; Barroso, G. C. ; Soares, J. M. Learning Vectors (LVs) um Instrumento Automatizado de Avaliação para Suporte a Aprendizagem em EaD. In: RENOTE Revista Novas Tecnologias na Educação. Julho 2008 V6 N1. CINTED/UFRGS Porto Alegre -RS.

Zabala, A. A Prática Educativa: como ensinar. Trad. Ernani F. da F. Rosa. Porto Alegre: ArtMed. 1998. 